PROCEEDINGS OF THE

AMERICAN MATHEMATICAL SOCIETY

Volume 139, Number 2, February 2011, Pages 641-653

S 0002-9939(2010)10559-2

Article electronically published on September 23, 2010

\title{
ON DEFORMATIONS OF COMPACT BALANCED MANIFOLDS
}

\author{
ALBERTO SARACCO AND ADRIANO TOMASSINI
}

(Communicated by Franc Forstneric)

\begin{abstract}
We study suitable deformations of the complex structure on a compact balanced manifold $(M, J, g, \omega)$, naturally associated with cohomology classes in $H^{2}(M, \mathbb{R})$. We construct curves of balanced structures on a compact holomorphically parallelizable 5-dimensional complex nilmanifold and of halfflat structures on a compact complex 3-dimensional solvmanifold respectively.
\end{abstract}

\section{INTRODUCTION}

Let $(M, J)$ be an $n$-dimensional complex manifold. A Hermitian metric $g$ on $(M, J)$ is said to be balanced (see [16]) if

$$
d \omega^{n-1}=0 \text {, }
$$

where $\omega(\cdot, \cdot)=g(J \cdot, \cdot)$ is the fundamental 2-form of $g$. Equivalently, $g$ is balanced if $\omega$ is co-closed, namely $d^{*} \omega=0$, where $d^{*}$ is the codifferential of $d$. The pair $(J, g)$, where $J$ is a complex structure and $g$ is a $J$-Hermitian balanced metric on $M$ respectively, is a balanced structure on $M$. We will refer to $(M, J, g, \omega)$ as a balanced manifold.

In the terminology of A. Gray and L. M. Hervella (see [10), a Hermitian metric $g$ on a $2 n$-dimensional almost complex manifold $(M, J)$ is said to be semi-Kähler if the fundamental form $\omega$ satisfies $d \omega^{n-1}=0$.

The existence of a balanced metric on an $n$-dimensional compact complex manifold $(M, J)$ has been intrinsically characterized by M. L. Michelsohn in terms of currents (see [16, Thm. 4.7]). For construction of balanced structures see also e.g. 9, 19 and the references therein.

The class of compact balanced manifolds have the nice property of being invariant under modifications. More precisely, in 2, 3, L. Alessandrini and G. Bassanelli showed that if $\tilde{M}$ and $M$ are compact complex manifolds and $f: \tilde{M} \rightarrow M$ is a modification, then $\tilde{M}$ is balanced if and only if $M$ is balanced. In particular, modifications of compact Kähler manifolds are always balanced.

In contrast with the Kähler case, compact balanced manifolds are not stable under small deformations of the complex structure (see [1, Prop. 4.1]). Therefore, given a compact balanced manifold, it is natural to look for those deformations of the complex structure which remain still balanced.

Received by the editors March 3, 2010.

2010 Mathematics Subject Classification. Primary 53C15; Secondary 32 G05.

Key words and phrases. Balanced metric, half-flat structure, deformation.

This work was supported by the Project M.I.U.R. "Geometric Properties of Real and Complex Manifolds" and by G.N.S.A.G.A. of I.N.d.A.M.

(C)2010 American Mathematical Society 641

Reverts to public domain 28 years from publication 
To such a purpose, one can select suitable deformations of the complex structure on a compact balanced manifold $(M, J, g, \omega)$ naturally associated with cohomology classes and giving rise to curves of balanced structures. More precisely, starting with a balanced manifold $(M, J, g, \omega)$, we consider cohomology classes $[\psi] \in H^{2}(M, \mathbb{R})$, where $\psi$ is the harmonic representative of the class such that there exists a curve of balanced structures $\left(J_{t}, g_{t}, \omega_{t}\right)$, for small real $t$, with $\omega_{t}=\omega+t \psi+o(t)$ and $J_{0}=J$.

The idea to associate curves of almost complex structures with cohomology classes has been used already by J. Lee in 13 in the context of $J$-holomorphic curves, by P. de Bartolomeis in [6] in relation to symplectic deformations of Kähler structures, and by P. de Bartolomeis and F. Meylan [7] in deformations of $C R$ structures. In particular, in [6] cohomology classes giving rise to curves of Kähler structures on a compact Kähler manifold $(M, J, g, \omega)$ are characterized.

The situation in the balanced case seems to be more complicated than in the Kähler one. In many cases there simply are no complex deformations such that the deformed structure is integrable.

However, it may happen that the deformation preserves some weaker but still interesting geometrical properties of the manifold, as for instance the half-flat one. According to [4], an SU(3)-structure $(J, \omega, \Psi)$ on a 6-dimensional (real) manifold $M$ is said to be half-flat if the non-degenerate 2-form $\omega$ and the complex volume form $\Psi$ satisfy the following conditions:

$$
d(\omega \wedge \omega)=0, \quad d(\mathfrak{R e} \Psi)=0 .
$$

It is known that hypersurfaces in 7-dimensional manifolds with exceptional holonomy $\mathbb{G}_{2}$ have a half-flat structure given by the restriction of the holonomy group representation. Conversely, starting with a half-flat manifold, if certain evolution equations admit a solution coinciding with the initial structure at time zero, then the construction can be reversed, giving rise to a manifold with holonomy contained in $\mathbb{G}_{2}$ (see [12]).

In this paper, after recalling the differential graded Lie algebras setup on complex manifolds, the general construction of curves of complex structures starting with real 2-cohomology classes and the basic facts on deformation theory of complex structures (see Sections 1] and 2), we will construct curves of balanced structures on a compact holomorphically parallelizable 5-dimensional complex nilmanifold $M$, obtained as a compact quotient of the 5-dimensional complex nilpotent group (see e.g. [17]):

$$
G=\left\{A \in \mathrm{GL}(4, \mathbb{C}) \mid A=\left(\begin{array}{cccc}
1 & z_{1} & z_{3} & z_{5} \\
0 & 1 & 0 & z_{2} \\
0 & 0 & 1 & z_{4} \\
0 & 0 & 0 & 1
\end{array}\right), \quad z_{j} \in \mathbb{C}, j=1, \ldots, 5\right\}
$$

It turns out that some but not all cohomology classes produce curves of balanced structures. Indeed, we have the following:

There is a 4-dimensional subspace $W \subsetneq H^{2}(M, \mathbb{R})$ such that the harmonic representative $\psi$ of every cohomology class in $W$ gives rise to a curve of balanced structures on $M$, whose fundamental forms are given by

$$
\omega_{t}=\omega+t \psi+o(t)
$$

(see Theorem 3.1 for the precise statement). 
Finally, in the last section we construct curves of half-flat structures deforming a balanced structure on a compact solvmanifold $N$ endowed with a complex structure. We obtain the following:

Theorem 4.1. The harmonic representative $\psi$ of every cohomology class in $H^{2}(N, \mathbb{R})$ gives rise to a curve of half-flat structures on $N$, whose fundamental forms are given by $\omega_{t}=\omega+t \psi+o(t)$.

\section{Complex structures and DGLA}

Let $(M, J)$ be an $n$-dimensional compact complex manifold. We recall the definition of the differential graded Lie algebra, DGLA for short, governing the small deformations of $J$. Let $\Omega_{J}^{0, p}(M)$ be the space of sections of the bundle $\Lambda_{J}^{0, q}(M)$ of $(0, q)$-forms on $(M, J)$. Denote by $\Gamma\left(M, \Lambda^{0, p}(M) \otimes T^{1,0} M\right)$ the space of sections of the vector bundle $\Lambda^{0, p}(M) \otimes T^{1,0} M$. Set

$$
\mathcal{A}_{p}= \begin{cases}\Gamma\left(M, \Lambda^{0, p}(M) \otimes T^{1,0} M\right), & \text { if } 0 \leq p \leq n, \\ 0 & \text { otherwise },\end{cases}
$$

and define

$$
\mathcal{A}=\bigoplus_{p \in \mathbb{Z}} \mathcal{A}_{p}
$$

The bracket $[[]$,$] and the \bar{\partial}_{J}$-operator are defined as follows.

Definition 1.1. The bracket

$$
[[,]]: \mathcal{A}_{p} \times \mathcal{A}_{q} \rightarrow \mathcal{A}_{p+q}
$$

is defined in the following way:

- For every pair $Z, W \in \mathcal{A}_{0}$ set

$$
[[Z, W]]=[Z, W]
$$

where [,] is the usual bracket between complex vector fields.

- For every $\varphi \in \mathcal{A}_{1}$ and $Z \in \mathcal{A}_{0}$, $[[\varphi, Z]]=-[[Z, \varphi]] \in \mathcal{A}_{1}$ is defined as

$$
[[\varphi, Z]](\bar{W})=[\varphi(\bar{W}), Z]+\frac{1}{2} \varphi([Z, \bar{W}]+i J[Z, \bar{W}]) .
$$

- For every $\varphi \in \mathcal{A}_{1},[[\varphi, \varphi]] \in \mathcal{A}_{2}$ is defined by

$$
[[\varphi, \varphi]](\bar{Z}, \bar{W})=2[\varphi(\bar{Z}), \varphi(\bar{W})]-2 \varphi([\varphi(\bar{Z}), \bar{W}]+[\bar{Z}, \varphi(\bar{W})]) .
$$

- For every $\varphi, \psi \in \mathcal{A}_{1}$, define $[[\varphi, \psi]] \in \mathcal{A}_{2}$ as

$$
[[\varphi, \psi]]=\frac{1}{2}([[(\varphi+\psi),(\varphi+\psi)]]-[[\varphi, \varphi]]-[[\psi, \psi]]) .
$$

- For every $\alpha \in \Omega_{J}^{0, p}(M), \beta \in \Omega_{J}^{0, q}(M), \varphi, \psi \in \mathcal{A}_{1}$, set

$$
\begin{aligned}
{[[\alpha \wedge \varphi, \beta \wedge \psi]]=} & \left.(-1)^{q} \alpha \wedge \beta \wedge[[\varphi, \psi]]+(-1)^{p}(\rceil_{\psi} \alpha\right) \wedge \beta \wedge \varphi \\
& \left.+\alpha \wedge(\rceil_{\varphi} \beta\right) \wedge \psi,
\end{aligned}
$$

where $\tau_{\varphi}$ is the skew-symmetric derivation of degree 1 of $\Omega^{0, *}(M)$ such that, for every smooth function $\left.f,(\rceil_{\varphi} f\right)(\bar{Z})=\varphi(\bar{Z})(f)$, and, for every $\alpha \in \Omega_{J}^{0,1}(M)$,

$\left(\neg_{\varphi} \alpha\right)(\bar{Z}, \bar{W})=\varphi(\bar{Z})(\alpha(\bar{W}))-\varphi(\bar{W})(\alpha(\bar{Z}))-\alpha([\varphi(\bar{Z}), \bar{W}]+[\bar{Z}, \varphi(\bar{W})])$.

- Finally, extend $[[]$,$] by bilinearity to any pair of elements of \mathcal{A}$. 
Definition 1.2. The $\bar{\partial}_{J}$-operator is the map

$$
\bar{\partial}_{J}: \mathcal{A}_{p} \rightarrow \mathcal{A}_{p+1}
$$

defined by:

i) For every $Z \in \mathcal{A}_{0}$, set

$$
\left(\bar{\partial}_{J} Z\right)(\bar{W})=\frac{1}{2}([\bar{W}, Z]-i J[\bar{W}, Z]),
$$

for every vector field $\bar{W}$ of type $(0,1)$.

ii) For every $\varphi \in \mathcal{A}_{1}$, set

$$
\left(\bar{\partial}_{J} \varphi\right)(\bar{Z}, \bar{W})=\left(\bar{\partial}_{J} \varphi(\bar{W})\right) \bar{Z}-\left(\bar{\partial}_{J} \varphi(\bar{Z})\right) \bar{W}-\varphi([\bar{Z}, \bar{W}]),
$$

for every pair of vector fields $\bar{Z}, \bar{W}$ of type $(0,1)$.

iii) Extend $\bar{\partial}_{J}$ to $\mathcal{A}_{p}$ by Leibniz rule, i.e.

$$
\bar{\partial}_{J}(\alpha \wedge \varphi)=\bar{\partial}_{J} \alpha \wedge \varphi+(-1)^{p} \alpha \wedge \bar{\partial}_{J} \varphi
$$

for every $\alpha \in \Omega_{J}^{0, p}(M), \varphi \in \mathcal{A}_{1}$.

Note that, for any $\varphi \in \mathcal{A}_{p}, \psi \in \mathcal{A}_{q}, \rho \in \mathcal{A}_{r}$, it can be checked that

1) $[[\varphi, \psi]]=-(-1)^{p q}[[\psi, \varphi]]$,

2) $[[\varphi,[[\psi, \rho]]]]=[[[[\varphi, \psi]], \rho]]+(-1)^{p q}[[\psi,[[\varphi, \rho]]]]$,

3) $\bar{\partial}_{J}^{2}=0$,

4) $\bar{\partial}_{J}[[\varphi, \psi]]=\left[\left[\bar{\partial}_{J} \varphi, \psi\right]\right]+(-1)^{p}\left[\left[\varphi, \bar{\partial}_{J} \psi\right]\right]$.

Therefore, $\left(\mathcal{A},[[]],, \bar{\partial}_{J}\right)$ is a DGLA.

Remark 1.3. Note that the theory can be set in real terms. To this purpose, let

$$
\begin{aligned}
\mathcal{A}_{p}^{\mathbb{R}} & =\left\{\varphi \in \Gamma\left(M, \Lambda^{p}(M) \otimes T M\right) \mid \varphi\left(X_{1}, \ldots, J X_{j}, \ldots, X_{p}\right)\right. \\
& \left.=-J \varphi\left(X_{1}, \ldots, X_{p}\right), \forall j=1, \ldots, p\right\}
\end{aligned}
$$

e.g. $\mathcal{A}_{0}^{\mathbb{R}}$ is the space of smooth vector fields and

$$
\mathcal{A}_{1}^{\mathbb{R}}=\{L \in \operatorname{End}(T M) \mid L J+J L=0\} .
$$

Let $m: T M \rightarrow T^{1,0} M$ be defined as

$$
m(X)=\frac{1}{2}(X-i J X) .
$$

Then every element $L \in \mathcal{A}_{p}^{\mathbb{R}}$ can be identified with a real form $m(L) \in \mathcal{A}_{p}$ by setting

$$
m(L)=\frac{1}{2}(L-i J L) .
$$

The inverse of $m$ is defined by

$$
m^{-1}(R)=R+\bar{R} .
$$

In particular, for every pair of vector fields $X, Y$,

$$
[[X, Y]]=m^{-1}[[m(X), m(Y)]]=\frac{1}{2}([X, Y]-[J X, J Y]),
$$

and for every vector field $X \in \mathcal{A}_{0}^{\mathbb{R}}$,

$$
\left(\bar{\partial}_{J} X\right)(Y)=\frac{1}{2}([Y, X]+J[J Y, X]),
$$


and for every $L \in \mathcal{A}_{1}^{\mathbb{R}}$

$$
\left(\bar{\partial}_{J} L\right)(X, Y)=\left(\bar{\partial}_{J} L(Y)\right)(X)-\left(\bar{\partial}_{J} L(X)\right)(Y)-L[[X, Y]],
$$

where we used the identification $\bar{\partial}_{J}=m^{-1} \circ \bar{\partial}_{J} \circ m$.

\section{Curves of Complex structures}

Let $(T, J, g)$ be a Hermitian vector space, i.e. a real vector space $T$ equipped with $J \in \operatorname{End}(T)$ satisfying $J^{2}=-I$ and a positive definite scalar product $g$ on $T$ satisfying $g(J X, J Y)=g(X, Y)$.

Let $\mathrm{V}$ be an endomorphism of $T$ anticommuting with $J$. We can identify $V$ with

$$
V: T^{0,1} \rightarrow T^{1,0}, \quad V(X+i J X)=\frac{1}{2}(V(X)-i J V(X)),
$$

which is $\mathbb{C}$-linear, where as usual

$$
T^{1,0}=\{X-i J X \mid X \in T\}, \quad T^{0,1}=\{X+i J X \mid X \in T\} .
$$

This identifies canonically the space

$$
\{V \in \operatorname{End}(T) \mid V J+J V=0\}
$$

with $\left(T^{*}\right)^{0,1} \otimes T^{1,0}$.

If, moreover, $V$ is antisymmetric, then, setting

$$
\alpha(X, Y)=g(V(X), Y),
$$

we obtain a two-form $\alpha \in \Lambda^{2,0+0,2} T^{*}$, and in terms of complexified space $\alpha=\gamma+\bar{\gamma}$, where

$$
\begin{gathered}
\gamma(X, Y)=\alpha^{2,0}(X, Y)=\frac{1}{2}(\alpha(X, Y)-i \alpha(J X, Y)), \\
\gamma \in \Lambda^{2,0} T^{*}, \quad \gamma(Z, W)=\overline{g(V(\bar{Z}), \bar{W})} .
\end{gathered}
$$

We will now recall the basic facts about the deformation theory of complex manifolds. Let $(M, J)$ be a compact complex manifold of real dimension $2 n$. On the space $\Gamma(M, \operatorname{End}(T M))$ of $\mathcal{C}^{\infty}$-sections of the vector bundle $\operatorname{End}(T M)=T^{*} M \otimes T M$ we will consider the usual topology. Then every almost complex structure $\hat{J}$ in a neighborhood of $J$ can be uniquely represented as

$$
\hat{J}=(I+L) J(I+L)^{-1},
$$

with $L \in \operatorname{End}(T M)$ such that $L J+J L=0$ (see e.g. [7). It turns out that $\hat{J}$ is integrable (see e.g. [15]) if and only if

$$
\hat{L}:=\frac{1}{2}(L-i J L) \in \mathcal{A}_{1}
$$

satisfies the following:

$$
\bar{\partial}_{J} \hat{L}+\frac{1}{2}[[\hat{L}, \hat{L}]]=0 .
$$

Equation (2.4) is called the Maurer-Cartan equation. Therefore the elements $L \in \mathcal{A}_{1}^{\mathbb{R}}$ such that $\operatorname{det}(I+L) \neq 0$ and $\hat{L}$ satisfies the Maurer-Cartan equation parametrize the complex structures close to $J$. More precisely, the map

$$
L \mapsto(I+L) J(I+L)^{-1}
$$


gives rise to a bijection between

$$
\left\{L \in \Omega_{J}^{0,1}(M)_{\mathbb{R}} \otimes T M \mid \operatorname{det}(I+L) \neq 0, \bar{\partial}_{J} \hat{L}+\frac{1}{2}[[\hat{L}, \hat{L}]]=0\right\}
$$

and

$$
\{\text { complex structures } \hat{J} \text { on } M\}
$$

in a neighborhood of $0 \in \Gamma(M, \operatorname{End}(T M))$ and in a neighborhood of $J$.

Let

$$
t \mapsto J_{t},
$$

be a smooth curve of almost complex structures on $M$, with $J_{0}=J$. Then, as just recalled above, for $-\epsilon<t<\epsilon$,

$$
J_{t}=\left(I-L_{t}\right) J\left(I-L_{t}\right)^{-1},
$$

where

$$
L_{t} J+J L_{t}=0, \quad L_{t}=t L+o(t) .
$$

It is immediate to check that

$$
\left.\frac{d}{d t} J_{t}\right|_{t=0}=2 L J
$$

Furthermore, if $t \mapsto J_{t}$ is a (smooth) curve of complex structures, then as a consequence of [7, Prop. 3.3], it follows that the linear map $L$ given by (2.5) satisfies the necessary condition

$$
\bar{\partial}_{J} L=0 .
$$

Let $[\gamma] \in H_{J}^{(2,0)+(0,2)}(M)_{\mathbb{R}}$ (see [14] and (2.6) $), \gamma$ being the harmonic representative, and let $V$ be as in (2.1). Then, defining

$$
\hat{L}=\frac{1}{2} V J
$$

one has that

$$
J_{t}=(I-t L) J(I-t L)^{-1}
$$

is a curve of almost complex structures on $M$.

In Sections 3 and 4 starting with the harmonic representatives of cohomology classes of type $(2,0)+(0,2)$, as in (2.2), and of type $(1,1)$ respectively, we will apply the previous constructions to deform balanced structures on two different compact non-Kähler manifolds.

Finally, we recall the definition of the cohomology groups (see [14])

$$
H_{J}^{(p, q)+(q, p)}(M)_{\mathbb{R}}=\left\{[\alpha] \mid \alpha \in \Omega_{J}^{p, q}(M) \oplus \Omega_{J}^{q, p}(M), \quad \alpha=\bar{\alpha}\right\},
$$

which will be used in the next sections. 


\section{BALANCED DEFORMATIONS}

Let $G \subset \mathrm{GL}(4, \mathbb{C})$ be the complex subgroup, with Lie algebra $\mathfrak{g}$, defined as

$$
G=\left\{A \in \operatorname{GL}(4, \mathbb{C}) \mid A=\left(\begin{array}{cccc}
1 & z_{1} & z_{3} & z_{5} \\
0 & 1 & 0 & z_{2} \\
0 & 0 & 1 & z_{4} \\
0 & 0 & 0 & 1
\end{array}\right), \quad z_{j} \in \mathbb{C}, j=1, \ldots, 5\right\} .
$$

Then $G$ is a complex nilpotent Lie group; the subset $\Gamma \subset G$ whose matrices have Gaussian integers entries is a uniform discrete subgroup, so that $M=\Gamma \backslash G$ is a compact 5-dimensional complex nilmanifold. The 1-forms

$$
\varphi^{j}=d z_{j}, \quad j=1, \ldots, 4, \quad \varphi^{5}=d z_{5}-z_{1} d z_{2}-z_{3} d z_{4}
$$

are $G$-invariant and, consequently, induce 1 -forms of type $(1,0)$ on $M$. Hence $M$ is holomorphically parallelizable, with structure equations

$$
d \varphi^{j}=0, \quad j=1, \ldots, 4, \quad d \varphi^{5}=-\varphi^{1} \wedge \varphi^{2}-\varphi^{3} \wedge \varphi^{4} .
$$

Denoting by $\left\{\theta_{1}, \ldots, \theta_{5}\right\}$ the global vector fields dual to $\left\{\varphi^{1}, \ldots, \varphi^{5}\right\}$, we immediately get that

$$
\left[\theta_{1}, \theta_{2}\right]=\theta_{5}, \quad\left[\theta_{3}, \theta_{4}\right]=\theta_{5}
$$

and the other brackets vanish. Setting $\varphi^{j}=e^{2 j-1}+i e^{2 j}, j=1, \ldots, 5$ and $\theta_{k}=$ $\frac{1}{2}\left(e_{2 k-1}-i e_{2 k}\right), k=1, \ldots, 5$, we have

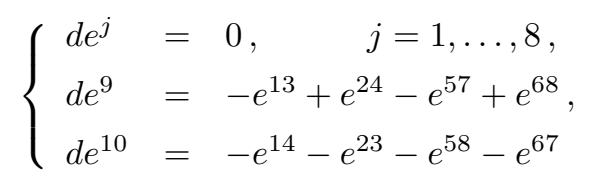

and

$$
\begin{aligned}
& {\left[e_{1}, e_{3}\right]=\left[e_{4}, e_{2}\right]=\left[e_{5}, e_{7}\right]=\left[e_{8}, e_{6}\right]=e_{9},} \\
& {\left[e_{1}, e_{4}\right]=\left[e_{2}, e_{3}\right]=\left[e_{5}, e_{8}\right]=\left[e_{6}, e_{7}\right]=e_{10},}
\end{aligned}
$$

the other brackets vanishing.

Observe that $M$ is a compact balanced manifold, the balanced form given by

$$
\omega=\frac{i}{2}\left(\varphi^{1} \wedge \bar{\varphi}^{1}+\varphi^{2} \wedge \bar{\varphi}^{2}+\varphi^{3} \wedge \bar{\varphi}^{3}+\varphi^{4} \wedge \bar{\varphi}^{4}+\varphi^{5} \wedge \bar{\varphi}^{5}\right)
$$

By Nomizu Theorem (see [18]) it is immediate to check that $b_{2}(M)=26$. Furthermore,

$$
H^{2}(M, \mathbb{R})=H^{(2,0)+(0,2)}(M)_{\mathbb{R}} \oplus H^{1,1}(M)_{\mathbb{R}} .
$$

One can check that $H^{(2,0)+(0,2)}(M)_{\mathbb{R}}, H^{1,1}(M)_{\mathbb{R}}$, respectively, are spanned by the following harmonic forms:

$$
\left\{\begin{array}{l}
\psi^{1}=\frac{1}{2}\left(\varphi^{13}+\varphi^{\overline{13}}\right), \psi^{2}=\frac{1}{2}\left(\varphi^{24}+\varphi^{\overline{24}}\right), \psi^{3}=\frac{1}{2}\left(\varphi^{14}+\varphi^{\overline{14}}\right), \\
\psi^{4}=\frac{1}{2}\left(\varphi^{23}+\varphi^{\overline{23}}\right), \psi^{5}=\frac{1}{2}\left(\varphi^{12}+\varphi^{\overline{12}}-\varphi^{34}-\varphi^{\overline{34}}\right), \\
\psi^{6}=\frac{1}{2 i}\left(\varphi^{12}-\varphi^{\overline{12}}-\varphi^{34}+\varphi^{\overline{34}}\right), \psi^{7}=\frac{1}{2 i}\left(\varphi^{14}-\varphi^{\overline{14}}\right), \\
\psi^{8}=\frac{1}{2 i}\left(\varphi^{23}-\varphi^{\overline{23}}\right), \psi^{9}=\frac{1}{2 i}\left(\varphi^{13}-\varphi^{\overline{13}}\right), \psi^{10}=\frac{1}{2 i}\left(\varphi^{24}-\varphi^{\overline{24}}\right),
\end{array}\right.
$$


and

$$
\left\{\begin{array}{l}
\psi^{11}=\frac{1}{2}\left(\varphi^{1 \overline{4}}+\varphi^{\overline{1} 4}\right), \psi^{12}=\frac{1}{2}\left(\varphi^{1 \overline{3}}+\varphi^{\overline{1} 3}\right), \psi^{13}=\frac{1}{2}\left(\varphi^{2 \overline{4}}+\varphi^{\overline{2} 4}\right), \\
\psi^{14}=\frac{1}{2}\left(\varphi^{2 \overline{3}}+\varphi^{\overline{2} 3}\right), \psi^{15}=\frac{i}{2} \varphi^{1 \overline{1}}, \psi^{16}=\frac{i}{2} \varphi^{2 \overline{2}}, \psi^{17}=\frac{i}{2} \varphi^{3 \overline{3}}, \\
\psi^{18}=\frac{i}{2} \varphi^{4 \overline{4}}, \psi^{19}=\frac{1}{2}\left(\varphi^{1 \overline{2}}+\varphi^{\overline{1} 2}\right), \psi^{20}=\frac{1}{2}\left(\varphi^{3 \overline{4}}+\varphi^{\overline{3} 4}\right), \\
\psi^{21}=\frac{1}{2 i}\left(\varphi^{1 \overline{2}}-\varphi^{\overline{1} 2}\right), \psi^{22}=\frac{1}{2 i}\left(\varphi^{1 \overline{3}}-\varphi^{\overline{1} 3}\right), \psi^{23}=\frac{1}{2 i}\left(\varphi^{1 \overline{4}}-\varphi^{\overline{1} 4}\right), \\
\psi^{24}=\frac{1}{2 i}\left(\varphi^{2 \overline{3}}-\varphi^{\overline{2} 3}\right), \psi^{25}=\frac{1}{2 i}\left(\varphi^{2 \overline{4}}-\varphi^{\overline{2} 4}\right), \psi^{26}=\frac{1}{2 i}\left(\varphi^{3 \overline{4}}-\varphi^{\overline{3} 4}\right),
\end{array}\right.
$$

where $\varphi^{\bar{h}}=\bar{\varphi}^{h}$ and $\varphi^{A B}=\varphi^{A} \wedge \varphi^{B}$. We can state the following.

Theorem 3.1. The harmonic representative $\psi$ of any cohomology class in

$$
\operatorname{Span}_{\mathbb{R}}\left\langle\psi^{1}, \psi^{2}, \psi^{3}, \psi^{4}\right\rangle \subset H^{2}(M, \mathbb{R})
$$

gives rise to a curve of balanced structures on $M$ whose fundamental forms are given by

$$
\omega_{t}=\omega+t \psi+o(t)
$$

Proof. Let

$$
\psi=\sum_{h=1}^{4} c_{h} \psi^{h} \in \operatorname{Span}_{\mathbb{R}}\left\langle\psi^{1}, \ldots, \psi^{4}\right\rangle
$$

We will show that there exists a curve of balanced structures $\left(J_{t}, \omega_{t}\right)$ on $M$ such that

$$
J_{0}=J,\left.\quad \frac{d}{d t} \omega_{t}\right|_{t=0}=\psi .
$$

Let $c_{1} \psi^{1} \in H^{(2,0)+(0,2)}(M)_{\mathbb{R}}$, where $\psi^{1}$ is defined as in (3.2). By (2.2), the linear map $V_{1}$ representing $\psi_{1}$ is given by

$$
V_{1}=\varphi^{\overline{1}} \otimes \theta_{3}-\varphi^{\overline{3}} \otimes \theta_{1}
$$

Then, setting $\hat{L}_{1}:=\frac{1}{2} V_{1} J$, we have

$$
\hat{L}_{1}=-\frac{i}{2}\left(\varphi^{\overline{1}} \otimes \theta_{3}-\varphi^{\overline{3}} \otimes \theta_{1}\right)
$$

and in real terms

$$
L_{1}=\hat{L}_{1}+\overline{\hat{L}}_{1}=-\frac{1}{2}\left(e^{1} \otimes e_{6}+e^{2} \otimes e_{5}-e^{5} \otimes e_{2}-e^{6} \otimes e_{1}\right) .
$$

Then $c_{1} L_{1}$ represents the $(2,0)+(0,2)$-form $c_{1} \psi^{1}$. We start by proving that $c_{1} L_{1}$ satisfies the Maurer-Cartan equation (2.4). In order to do this, we show that

$$
\begin{array}{ll}
\text { i) } & \bar{\partial}_{J} \hat{L}_{1}=0, \\
\text { ii) } & {\left[\left[\hat{L}_{1}, \hat{L}_{1}\right]\right]=0 .}
\end{array}
$$

By definition,

$$
\bar{\partial}_{J} \hat{L}_{1}\left(\bar{\theta}_{r}, \bar{\theta}_{s}\right)=\left(\bar{\partial}_{J} \hat{L}_{1}\left(\bar{\theta}_{s}\right)\right)\left(\bar{\theta}_{r}\right)-\left(\bar{\partial}_{J} \hat{L}_{1}\left(\bar{\theta}_{r}\right)\right)\left(\bar{\theta}_{s}\right)-\hat{L}_{1}\left(\left[\bar{\theta}_{r}, \bar{\theta}_{s}\right]\right) .
$$

Now observe that

$$
\hat{L}_{1}\left(\left[\bar{\theta}_{r}, \bar{\theta}_{s}\right]\right)=0
$$

since $\left[\bar{\theta}_{1}, \bar{\theta}_{2}\right]=\left[\bar{\theta}_{3}, \bar{\theta}_{4}\right]=\bar{\theta}_{5}$, the other brackets vanishing. Furthermore since

$$
\left[\theta_{r}, \bar{\theta}_{s}\right]=0, \quad r, s=1, \ldots, 5
$$


we obtain

$$
\bar{\partial}_{J} \hat{L}_{1}\left(\bar{\theta}_{r}, \bar{\theta}_{s}\right)=0 \text {. }
$$

We have

$$
\begin{aligned}
{\left[\left[\hat{L}_{1}, \hat{L}_{1}\right]\right]\left(\bar{\theta}_{r}, \bar{\theta}_{s}\right)=} & 2\left[\delta_{r}^{1} \theta_{3}-\delta_{r}^{3} \theta_{1}, \delta_{s}^{1} \theta_{3}-\delta_{s}^{3} \theta_{1}\right]-2 \hat{L}_{1}\left(\left[\delta_{r}^{1} \theta_{3}-\delta_{r}^{3} \theta_{1}, \bar{\theta}_{s}\right]\right. \\
& \left.+\left[\bar{\theta}_{r}, \delta_{s}^{1} \theta_{3}-\delta_{s}^{3} \theta_{1}\right]\right)=0,
\end{aligned}
$$

since again $\left[\theta_{1}, \theta_{3}\right]=0$ and $\left[\theta_{r}, \bar{\theta}_{s}\right]=0, r, s=1, \ldots, 5$. Consequently

$$
\bar{\partial}_{J} t c_{1} \hat{L}_{1}+\frac{1}{2}\left[\left[t c_{1} \hat{L}_{1}, t c_{1} \hat{L}_{1}\right]\right]=0,
$$

namely $t c_{1} \hat{L}_{1}$ satisfies the Maurer-Cartan equation (2.4). Then $J_{t, c_{1}}$ is integrable.

By a similar computation, one can prove that the almost complex structures $J_{t, c_{h}}$ induced by the form $c_{h} \psi^{h}$ are actually integrable, for $h=2,3,4$. Therefore, also the form $\sum_{h=1}^{4} c_{h} \psi^{h}$ gives rise to a complex structure. Hence, if $L=\frac{1}{2} V J$, then

$$
J_{t}=(I-t L) J(I-t L)^{-1}
$$

is a curve of complex structures on $M$.

Set

$$
\varphi_{t}^{j}=e^{2 j-1}+i J_{t} e^{2 j-1},
$$

for $j=1, \ldots, 5$. Then define

$$
\omega_{t}=\frac{1}{2}\left(\omega+J_{t} \omega\right) .
$$

By definition, the real 2 -form $\omega_{t}$ is of type $(1,1)$ with respect to $J_{t}$, and, for small $t$, it is strictly positive. A direct computation shows that

$$
d \omega_{t}^{4}=0,\left.\quad \frac{d}{d t} \omega_{t}\right|_{t=0}=\psi .
$$

Therefore $\left(J_{t}, \omega_{t}\right)$ is a curve of balanced structures induced by $\psi$.

\section{HalF-Flat DEFORMATiOnS}

In this section we will provide a 6 -dimensional balanced manifold $(N, J, g, \omega)$ which has half-flat deformations in every direction. We first recall the construction of $N$ (see [17 and [8]).

Let $x_{1}, \ldots, x_{6}$ denote coordinates on $\mathbb{R}^{6}$.

We set $\lambda=\log \frac{3+\sqrt{5}}{2}$ and $\mu=\frac{1-\sqrt{5}}{2}$ and consider the following transformation of $\mathbb{R}^{6}$ :

$$
T_{1}\left(x_{1}, x_{2}, x_{3}, x_{4}, x_{5}, x_{6}\right)=\left(x_{1}+\lambda, e^{-\lambda} x_{2}, e^{\lambda} x_{3}, x_{4}, e^{-\lambda} x_{5}, e^{\lambda} x_{6}\right) .
$$

We set

$$
N=\frac{\mathbb{R}_{x_{4}}}{2 \pi \mathbb{Z}} \times \frac{\mathbb{R}_{x_{1}} \times \mathbb{R}_{x_{2}, x_{3}, x_{5}, x_{6}}^{4} / \Gamma}{\left\langle T_{1}(x)\right\rangle}
$$

where

$$
\Gamma=\operatorname{Span}_{\mathbb{Z}}\left\langle(\mu, 1,0,0)^{t},(1,-\mu, 0,0)^{t},(0,0,2 \pi \mu, 2 \pi)^{t},(0,0,2 \pi,-2 \pi \mu)^{t}\right\rangle
$$


and $\left\langle T_{1}(x)\right\rangle$ denotes the subgroup of transformations generated by $T_{1}(x)$. Then $N$ is a compact 6-dimensional manifold. The six 1-forms on $\mathbb{R}^{6}$,

$$
\left\{\begin{array}{l}
e^{1}=d x_{1}, \\
e^{2}=\exp \left(x_{1}\right) d x_{2}, \\
e^{3}=\exp \left(-x_{1}\right) d x_{3}, \\
e^{4}=d x_{4} \\
e^{5}=\exp \left(x_{1}\right) d x_{5}, \\
e^{6}=\exp \left(-x_{1}\right) d x_{6},
\end{array}\right.
$$

induce 1-forms on the manifold $N$. Therefore, we immediately get

$$
\left\{\begin{array}{l}
d e^{1}=0, \\
d e^{2}=e^{1} \wedge e^{2}, \\
d e^{3}=-e^{1} \wedge e^{3}, \\
d e^{4}=0 \\
d e^{5}=e^{1} \wedge e^{5}, \\
d e^{6}=-e^{1} \wedge e^{6} .
\end{array}\right.
$$

In fact it turns out that $N$ can also be presented as a compact quotient of a 6 dimensional simply connected completely solvable Lie group by a uniform discrete subgroup (see [5] and [11, Example 5]).

Let us consider the complex structure on $N$ defined by saying that the following $\varphi^{j}=e^{j}+i e^{j+3}, j=1,2,3$, are the complex $(1,0)$-forms. Then one has

$$
\left\{\begin{array}{l}
d \varphi^{1}=0, \\
d \varphi^{2}=e^{12}+i e^{15}=\frac{1}{2}\left(\varphi^{1}+\bar{\varphi}^{1}\right) \wedge \varphi^{2}, \\
d \varphi^{3}=-e^{13}-i e^{16}=-\frac{1}{2}\left(\varphi^{1}+\bar{\varphi}^{1}\right) \wedge \varphi^{3} .
\end{array}\right.
$$

Observe that $N$ is a compact balanced manifold, with balanced form given by

$$
\omega=\frac{i}{2}\left(\varphi^{1} \wedge \bar{\varphi}^{1}+\varphi^{2} \wedge \bar{\varphi}^{2}+\varphi^{3} \wedge \bar{\varphi}^{3}\right) .
$$

A basis of $H^{2}(N, \mathbb{R})$ is given by

$$
\begin{gathered}
H^{1,1}(N)_{\mathbb{R}} \ni\left\{\begin{array}{l}
\psi^{1}=\frac{i}{2} \varphi^{1} \wedge \bar{\varphi}^{1}, \\
\psi^{2}=\frac{1}{2}\left(\varphi^{2} \wedge \bar{\varphi}^{3}+\bar{\varphi}^{2} \wedge \varphi^{3}\right), \\
\psi^{3}=\frac{i}{2}\left(\varphi^{2} \wedge \bar{\varphi}^{3}-\bar{\varphi}^{2} \wedge \varphi^{3}\right),
\end{array}\right. \\
H^{(2,0)+(0,2)}(N)_{\mathbb{R}} \ni\left\{\begin{array}{l}
\psi^{4}=-\frac{i}{2}\left(\varphi^{2} \wedge \varphi^{3}-\bar{\varphi}^{2} \wedge \bar{\varphi}^{3}\right), \\
\psi^{5}=\frac{1}{2}\left(\varphi^{2} \wedge \varphi^{3}+\bar{\varphi}^{2} \wedge \bar{\varphi}^{3}\right),
\end{array}\right.
\end{gathered}
$$

where $\psi^{j}, j=1, \ldots, 5$, are harmonic with respect to the metric $g=\sum_{i=1}^{6} e^{i} \otimes e^{i}$. We have the following: 
Theorem 4.1. The harmonic representative $\psi$ of any cohomology class in $H^{2}(N, \mathbb{R})$ gives rise to a curve of half-flat structures on $N$ whose fundamental forms are given by $\omega_{t}=\omega+t \psi+o(t)$.

Proof. In view of (4.1) and (4.2), if $\psi$ is a $g$-harmonic 2 -form on $N$, then we can write

$$
\psi=\sum_{j=1}^{5} c_{j} \psi^{j},
$$

where $c_{j} \in \mathbb{R}$.

We first deform the balanced form $\omega$ in the direction of the $(2,0)+(0,2)$ part of $\psi$.

The linear map $V: T^{0,1} N \rightarrow T^{1,0} N$ associated with $c_{4} \psi^{4}+c_{5} \psi^{5}$ is given by

$$
V=\left(c_{5}+i c_{4}\right)\left(\bar{\varphi}^{2} \otimes \theta_{3}-\bar{\varphi}^{3} \otimes \theta_{2}\right) .
$$

Hence, setting $\hat{L}=\frac{1}{2} V J$, we get

$$
\hat{L}=\frac{1}{2}\left(c_{4}-i c_{5}\right)\left(\bar{\varphi}^{2} \otimes \theta_{3}-\bar{\varphi}^{3} \otimes \theta_{2}\right) .
$$

In real terms,

$$
L=-\frac{1}{2} c_{4}\left(-e^{2} \otimes e_{3}+e^{5} \otimes e_{6}+e^{3} \otimes e_{2}-e^{6} \otimes e_{5}\right)-\frac{1}{2} c_{5}\left(e^{2} \otimes e_{6}+e^{5} \otimes e_{3}-e^{6} \otimes e_{2}-e^{3} \otimes e_{5}\right) .
$$

Thus is defined the curve of (not integrable unless $c_{4}=c_{5}=0$ ) almost complex structures

$$
J_{t}=(I-t L) J(I-t L)^{-1} .
$$

The almost complex structure $J_{t}$ defines a deformation of the form $\varphi^{1}, \varphi^{2}, \varphi^{3}$ as follows:

$$
\left\{\begin{array}{l}
\varphi_{t}^{1}=e^{1}+i J_{t} e^{1}=e^{1}+i e^{4}, \\
\varphi_{t}^{2}=e^{2}+i J_{t} e^{2}=e^{2}+i \frac{1}{f\left(t, c_{4}, c_{5}\right)}\left[4 c_{5} t e^{3}+\left(4-c_{4}^{2} t^{2}-c_{5}^{2} t^{2}\right) e^{5}+4 c_{4} t e^{6}\right], \\
\varphi_{t}^{3}=e^{3}+i J_{t} e^{3}=e^{3}+i \frac{1}{f\left(t, c_{4}, c_{5}\right)}\left[-4 c_{5} t e^{2}-4 c_{4} t e^{5}+\left(4-c_{4}^{2} t^{2}-c_{5}^{2} t^{2}\right) e^{6}\right],
\end{array}\right.
$$

where $f\left(t, c_{4}, c_{5}\right)=c_{4}^{2} t^{2}+c_{5}^{2} t^{2}+4$. We set

$$
\psi_{t}^{1}=\frac{i}{2} \varphi_{t}^{1} \wedge \bar{\varphi}_{t}^{1}, \psi_{t}^{2}=\frac{1}{2}\left(\varphi_{t}^{2} \wedge \bar{\varphi}_{t}^{3}+\bar{\varphi}_{t}^{2} \wedge \varphi_{t}^{3}\right), \psi_{t}^{3}=\frac{i}{2}\left(\varphi_{t}^{2} \wedge \bar{\varphi}_{t}^{3}-\bar{\varphi}_{t}^{2} \wedge \varphi_{t}^{3}\right) .
$$

Note that $\psi_{0}^{j}=\psi^{j}$, for $j=1,2,3$.

We define a family of SU(3)-structures on $N$. Set

$$
\omega_{t}=\frac{i}{2}\left(\varphi_{t}^{1} \wedge \bar{\varphi}_{t}^{1}+\varphi_{t}^{2} \wedge \bar{\varphi}_{t}^{2}+\varphi_{t}^{3} \wedge \bar{\varphi}_{t}^{3}\right)+t\left(c_{1} \psi_{t}^{1}+\left(c_{2}-c_{5}\right) \psi_{t}^{2}+c_{3} \psi_{t}^{3}\right) .
$$

The form $\omega_{t}$ is of type $(1,1)$ with respect to $J_{t}$. It is immediate to check that the tangent direction of $\omega_{t}$ at $t=0$ is indeed $\psi$. A straightforward computation yields

$$
d\left(\omega_{t} \wedge \omega_{t}\right)=0 .
$$

Let $\hat{\Psi}_{t}=\varphi_{t}^{1} \wedge \varphi_{t}^{2} \wedge \varphi_{t}^{3}$. Then, up to rescaling $\hat{\Psi}$, we may assume that

$$
\hat{\Psi}_{t} \wedge \overline{\hat{\Psi}}_{t}=-8 i \frac{\omega_{t}^{3}}{3 !} .
$$


Therefore, $\left(J_{t}, \omega_{t}, \hat{\Psi}_{t}\right)$ gives rise to a family of $\mathrm{SU}(3)$-structures on $N$. We will show that there exists a family of complex numbers $a\left(t, c_{4}, c_{5}\right)+i b\left(t, c_{4}, c_{5}\right) \in \mathbb{S}^{1}$ such that, setting

$$
\Psi_{t}=\left(a\left(t, c_{4}, c_{5}\right)+i b\left(t, c_{4}, c_{5}\right)\right) \hat{\Psi}_{t},
$$

$\left(J_{t}, \omega_{t}, \Psi_{t}\right)$ is a family of $\mathrm{SU}(3)$-structures on $N$ satisfying

$$
d\left(\mathfrak{R e} \Psi_{t}\right)=0 ;
$$

namely, in view of (4.3), $\left(J_{t}, \omega_{t}, \Psi_{t}\right)$ is a curve of half-flat structures on $N$. We easily obtain

$$
\begin{aligned}
d\left(\mathfrak{R e} \hat{\Psi}_{t}\right) & =8 \frac{c_{4} t}{f\left(t, c_{4}, c_{5}\right)}\left(e^{1346}-e^{1245}\right)=B\left(t, c_{4}, c_{5}\right)\left(e^{1346}-e^{1245}\right) \\
d\left(\Im \mathfrak{m} \hat{\Psi}_{t}\right) & =8 \frac{c_{5} t\left(f\left(t, c_{4}, c_{5}\right)-8\right)}{f\left(t, c_{4}, c_{5}\right)^{2}}\left(e^{1346}-e^{1245}\right)=A\left(t, c_{4}, c_{5}\right)\left(e^{1346}-e^{1245}\right) .
\end{aligned}
$$

Setting

then

$$
a\left(t, c_{4}, c_{5}\right)=\frac{A}{\sqrt{A^{2}+B^{2}}}, \quad b\left(t, c_{4}, c_{5}\right)=\frac{B}{\sqrt{A^{2}+B^{2}}},
$$

The proof is complete.

$$
d\left(\mathfrak{R e} \Psi_{t}\right)=0, \quad \Psi_{t} \wedge \bar{\Psi}_{t}=-8 i \frac{\omega_{t}^{3}}{3 !} .
$$

\section{REFERENCES}

[1] L. Alessandrini, G. Bassanelli, Small deformations of a class of compact non-Kähler manifolds, Proc. Amer. Math. Soc. 109 (1990), 1059-1062. MR.1012922 (90k:32064)

[2] _ Metric properties of manifolds bimeromorphic to compact Kähler spaces, J. Differential Geom. 37 (1993), 95-121. MR.1198601 (93m:32040)

[3] _ The Class of Compact Balanced Manifolds Is Invariant under Modifications, Complex Analysis and Geometry, Lecture Notes in Pure and Applied Mathematics, Marcel Dekker, 173, 1995, 1-17. MR.1365967 (96m:32030)

[4] S. Chiossi, S. M. Salamon, The intrinsic torsion of $\mathrm{SU}(3)$ and $G_{2}$ structures. Differential geometry, Valencia, 2001, 115-133, World Sci. Publ., River Edge, NJ, 2002. MR.1922042 (2003g:53030)

[5] L. C. de Andrés, M. Fernández, M. de León, J. J. Mencía, Some six-dimensional compact symplectic and complex solvmanifolds, Rend. Mat. Appl. (7) 12 (1992), 59-67. MR1186146 (93h:53030)

[6] P. de Bartolomeis, Symplectic deformations of Kähler manifolds, J. Symplectic Geom. 3 (2005), 341-355. MR2198780 (2006j:32022)

[7] P. de Bartolomeis, F. Meylan, Intrinsic deformation theory of $C R$ structures, Institut MittagLeffler, Report No. 12, 2007/2008, spring, to appear in Ann. Sc. Norm. Super. Pisa Cl. Sc.

[8] P. de Bartolomeis, A. Tomassini, On solvable generalized Calabi-Yau manifolds, Ann. Inst. Fourier 56 (2006), 1281-1296. MR2273857 (2007j:53105)

[9] M. Fernández, A. Tomassini, L. Ugarte, R. Villacampa, Balanced Hermitian metrics from SU(2)-structures, J. Math. Phys. 50 (2009), 15 pp. MR2510912 (2010f:53124)

[10] A. Gray, L. M. Hervella, The sixteen classes of almost Hermitian manifolds and their linear invariants, Ann. Mat. Pura e App. 123 (1980), 35-58. MR.581924 (81m:53045)

[11] K. Hasegawa, Complex and Kähler structures on compact solvmanifolds, J. Symplectic Geom. 3 (2005), 749-767. MR 2235860 (2007f:32026)

[12] N. Hitchin, Stable forms and special metrics, In Global Differential Geometry: The Mathematical Legacy of Alfred Gray, volume 288 of Contemp. Math., pages 70-89. American Math. Soc., 2001. MR1871001 (2003f:53065)

[13] J. Lee, Family Gromov-Witten invariants for Kähler surfaces, Duke. Math. J. 123 (2004), 209-233. MR2060027(2005d:53141) 
[14] T.-J. Li, W. Zhang, Comparing tamed and compatible symplectic cones and cohomological properties of almost complex manifolds, Comm. Anal. Geom. 17 (2009), 651-683. MR2601348

[15] J. Morrow, K. Kodaira, Complex Manifolds. Reprint of the 1971 edition with errata. AMS Chelsea Publishing, Providence, RI, 2006. MR2214741|(2006j:32001)

[16] M. L. Michelsohn, On the existence of special metrics in complex geometry, Acta Math. 143 (1983), 261-295. MR688351 (84i:53063)

[17] I. Nakamura, Complex parallelisable manifolds, J. Differential Geom. 10 (1975), 85-112. MR0393580 (52:14389)

[18] K. Nomizu, On the cohomology of compact homogeneous spaces of nilpotent Lie groups, Ann. of Math. (2) 59 (1954), 531-538. MR0064057 (16:219c)

[19] L. Ugarte, Hermitian structures on six dimensional nilmanifolds, Transfor. Groups. 12 (2007), 175-202. MR2308035 (2008e:53139)

Dipartimento di Matematica, Università di Parma, Parco Area delle Scienze 53/A, I-43124 PARMa, ITALY

E-mail address: alberto.saracco@unipr.it

Dipartimento di Matematica, Università di Parma, Parco Area delle Scienze 53/A, I-43124 PARMA, ITALY

E-mail address: adriano.tomassini@unipr.it 